\title{
1 Fungal communities in Scandinavian lakes along a longitudinal gradient
}

2 Maryia Khomich $^{1,2^{*}}$, Marie L. Davey ${ }^{2}$, Håvard Kauserud ${ }^{2}$, Serena Rasconi $^{3}$, Tom Andersen ${ }^{1}$

3

$4{ }^{1}$ Section for Aquatic Biology and Toxicology, Department of Biosciences, University of Oslo,

5 P.O. Box 1066 Blindern, 0316 Oslo, Norway

$6{ }^{2}$ Section for Genetics and Evolutionary Biology, Department of Biosciences, University of Oslo,

7 P.O. Box 1066 Blindern, 0316 Oslo, Norway

$8 \quad{ }^{3}$ WasserCluster-Biological Station Lunz, Inter-university Centre for Aquatic Ecosystem

9 Research, A-3293 Lunz am See, Austria

10

11 *Corresponding author: Department of Biosciences, University of Oslo, P.O. Box 1066

12 Blindern, 0316 Oslo, Norway

13 Tel.: +47-22845979, Fax: +47-22854726

14 E-mail address: maryia.khomich@ibv.uio.no, marykhomich@gmail.com

15

16 Running title: Fungal diversity in oligotrophic lakes

17 
19 This study investigates the diversity and distribution of fungal communities in 77 oligotrophic

20 lakes in southern Norway and Sweden using 454-sequencing with fungal-specific primers

21 targeting ITS2 region of the rRNA gene. A total of 232 operational taxonomic units (OTUs)

22 belonging to four recognized phyla were detected. A large proportion (70.69\%) of the detected

23 OTUs was Dikarya (Ascomycota and Basidiomycota), while Chytridiomycota dominated

24 quantitatively (63.37\% reads). The most abundant aquatic fungi were taxonomically assigned to

25 Chytridiomycota, whose members are known to be saprobes on a large variety of substrates and

26 parasites of phytoplankton, zooplankton, fungi and invertebrates, suggesting that resident fungi

27 strictly depend on surfaces and, therefore, are closely associated with other types of aquatic

28 organisms. Our results indicate that surface waters of oligotrophic lakes harbour a diverse pool

29 of fungi, both with tentative terrestrial and true aquatic origin. Longitude and environmental

30 factors were important in structuring the fungal community composition.

31

32 Keywords: aquatic fungi, Chytridiomycota, ITS region, freshwater, diversity, 454

33 pyrosequencing

34 


\section{Introduction}

36 Fungi are an ecologically and functionally diverse kingdom of eukaryotic organisms that have

37 evolved a wide array of mutualists (e.g. lichens, mycorrhizal fungi, endophytic fungi), parasites

38 and saprotrophs. Fungal communities have been shown to be both large and highly diverse in

39 soils and plant-associated habitats (Blaalid et al., 2012; Botnen et al., 2014; Tedersoo et al.,

40 2014). However, the current known biodiversity of aquatic fungi is poorly documented and the

41 number of aquatic species constitutes only a tiny fraction of those reported from terrestrial

42 environments. Nevertheless, fungi are common inhabitants of aquatic ecosystems and act as key

43 players in the turnover of both allochthonous and autochthonous organic matter, serving as

44 important mediators of energy and nutrient transfer to higher trophic levels (Kuehn, 2016).

45 Freshwater fungi are a taxonomically and morphologically diverse group found in various

46 aquatic habitats including lakes, ponds, rivers, streams, sediments, submerged substrata,

47 freshwater algae and invertebrates, as has been demonstrated by diversity studies from

48 temperate, tropical and subtropical regions (Duarte et al., 2016; Hyde et al., 2016). Additionally,

49 fungal sequences detected in aquatic environments span a large variety of novel, deep-branching,

50 and yet uncultured, fungal lineages, which have been termed 'dark matter fungi' (Grossart et al.,

51 2016).

52 Freshwater ecosystems have traditionally been subdivided into lentic (standing waters: lakes,

53 ponds, wetlands) and lotic (running waters: streams, rivers). In contrast to well-studied lotic

54 systems (Duarte et al., 2015; Graça et al., 2016), where fungi are mainly recognized as litter

55 decomposers (Duarte et al., 2015), lentic freshwater fungal diversity is only starting to be

56 unveiled using high-throughput sequencing (Comeau et al., 2016; Monchy et al., 2011), which

57 has identified a large number of unknown fungal lineages (Ishida et al., 2015). Lakes, the biggest 
freshwater reservoirs, are structured into littoral and pelagic zones. The former is a 'hotspot' for

59 all kinds of fungi providing diverse ecological niches, whereas the latter can both harbour highly specialized species and serve as a medium for propagule dispersal (Wurzbacher et al., 2010). The

61 Chytridiomycota, an early divergent fungal lineage, represents the best studied aquatic fungal

62 group, and occurs primarily in lakes where they are well adapted to the aquatic lifestyle, acting

63 both as saprotrophs and parasites of a wide range of hosts (Kagami et al., 2007; Kagami et al.,

64 2014; Rasconi et al., 2012; Sime-Ngando, 2012; Wurzbacher et al., 2014). Parasitism by chytrids

65 is an important ecological driving force in the aquatic food web dynamics (Rasconi et al., 2012;

66 Sime-Ngando, 2012). The transfer of nutrients from phytoplankton to zooplankton occurs via the

67 zoospores of parasitic chytrids through the 'mycoloop' (Kagami et al., 2014). In addition, other

68 possible mycoloops may exist in freshwater food webs, with saprotrophic chytrid zoospores

69 released from pollen and consumed by zooplankton (Kagami et al., 2014). Aquatic

70 hyphomycetes are common inhabitants of lakes (Chauvet et al., 2016; Wurzbacher et al., 2010).

71 Filamentous fungi that require solid substrata are widespread in the littoral zone of lakes where

72 there is substantial leaf litter input from the terrestrial vegetation (Wurzbacher et al., 2010).

73 Some studies to date suggest that yeast forms appear to dominate the known diversity of aquatic

74 fungi in the pelagic zone of lakes, as well as in marine environments (Bass et al., 2007; Richards

75 et al., 2012; Richards et al., 2015; Tisthammer et al., 2016). However, this view contradicts with

76 recent surveys in freshwater and marine ecosystems (Comeau et al., 2016; Hassett et al., 2016;

77 Hassett and Gradinger, 2016) reporting the dominance of Chytridiomycota.

78 The number of truly aquatic lichens is small, with 270 lichen and lichenicolous species occurring

79 regularly in freshwater of an estimated total of 13500 lichens (Jones et al., 2014; Thüs et al., 80 2014). Terrestrial filamentous fungi (e.g. endophytic and air-borne fungi) are often passively 
81 introduced into lakes in the form of spores and fragments of mycelia via inflowing streams,

82 rainwater, wind and soil particles (Voronin, 2014). However, it is often unclear whether such

83 fungi are terrestrial or truly aquatic (Wurzbacher et al., 2010). In most cases, the minimal

84 abundance of the spores of the terrestrial fungi is in the middle water layer and the maximal is

85 near the bottom, where fungal propagules accumulate during sedimentation (Voronin, 2014).

86 Different factors have been shown to influence the community composition of freshwater and

87 marine fungi. For example, temperature, $\mathrm{pH}$, conductivity, altitude, differences in the riparian

88 vegetation, seasonality in the temperate regions and the presence of various pollutants affected

89 the diversity and distribution of aquatic hyphomycetes (Duarte et al., 2016). In contrast, sample

90 depth, oxygen and nitrate concentrations explained $73 \%$ of the total variance in comparison to

$9118 \%$ explained by geographic location for marine fungal communities on a global scale

92 (Tisthammer et al., 2016).

93 In this study, we aimed to investigate the diversity and abundance of freshwater fungi in the 94 epilimnion of 77 ultra-oligotrophic to mesotrophic boreal lakes (Fig. 1) over a $750 \mathrm{~km}$ 95 longitudinal diversity gradient across southern Scandinavia (Ptacnik et al., 2010; Ptacnik et al., 96 2008) using the internal transcribed spacer (ITS2) marker. These boreal lakes represent a good 97 model to study compositional variation from a perspective of multiple communities connected by 98 dispersing organisms (Hortal et al., 2014; Leibold et al., 2004), with species richness in a given 99 site strongly linked to metacommunity dynamics and dispersal from adjacent sites (Ptacnik et al., 100 2010). Ptacnik et al. (2010) assessed the relative importance of local versus regional factors as 101 predictors of local genus richness in unicellular phytoplankton across Scandinavian lakes and 102 showed that phytoplankton metacommunities integrated richness of local communities across 103 environmental gradients on a scale between 100 and $400 \mathrm{~km}$. However, the Scandinavian 
104 diversity gradient is complex and not fully resolved as it coincides both with major changes in 105 landscape productivity, altitude and soil depth, as well as the main dispersal routes for freshwater 106 organisms after the glacial retreat (Khomich et al., in press). Recurring glaciations in boreal areas 107 can be considered an important, though neglected, historical climatic factor influencing biota 108 (Soininen, 2012). Lakes for our study were carefully selected to be as similar as possible with 109 respect to properties other than longitudinal position and local productivity (Table S1). Our 110 objectives were as follows: (i) to analyse taxonomic composition of aquatic fungal communities 111 across a known biodiversity gradient, (ii) to characterise the ecology of the detected fungal taxa 112 hypothesizing that both resident and transient components of aquatic communities are 113 simultaneously present, (iii) to explore the patterns of variation in fungal OTU composition 114 across lakes in this gradient to confirm whether it follows the same longitudinal pattern, as has 115 earlier been shown for phyto- and zooplankton diversity with non-molecular methods (Hessen et 116 al., 2006; Ptacnik et al., 2010) and 18S rDNA amplicon sequencing of eukaryotic communities in 117 these lakes (Khomich et al., in press).

\section{2. Materials and methods}

120 2.1. Site description

121 Lakes for this study were selected from the 'Rebecca' (Solheim et al., 2008) and 'Nordic lake 122 survey 1995' (Henriksen et al., 1998) data sets on Norwegian and Swedish lakes to generate a 123 subset of lakes fulfilling the following criteria: longitude $5-18{ }^{\circ} \mathrm{E}$, latitude $58-62{ }^{\circ} \mathrm{N}$, altitude $124<600 \mathrm{~m}$, surface area $>1 \mathrm{~km}^{2}$, total phosphorus $(\mathrm{TP})<30 \mu \mathrm{g} \mathrm{L}^{-1}$, total organic carbon (TOC) $125<30 \mathrm{mg} \mathrm{L}^{-1}$ and $\mathrm{pH}>5$. The lakes were chosen to create a representative subset of boreal lakes 126 with best possible coverage and orthogonality with respect to gradients of TP, TOC and 
127 longitudinal position. The former two represent two major effects on aquatic productivity

128 (Thrane et al., 2014), while the latter reflects the regional diversity gradient (Ptacnik et al., 129 2010). The three gradient variables were split in two factor levels (high/low), giving eight 130 different combinations of TP, TOC and longitude. A total of 12 lakes were randomly sampled 131 from each of the eight combinations. Sampling was performed mainly by hydroplane in July to 132 August 2011 (Thrane et al., 2014). Because of unfavorable weather conditions during sampling 133 the number of sampled lakes was eventually reduced to 77 (Fig.1).

\subsection{Sampling program}

136 Water samples were collected from the lake epilimnion $(0-5 \mathrm{~m})$ using an integrating water 137 sampler (Hydro-BIOS, Germany) in the central part of each lake during daytime. For DNA 138 analysis, up to $15 \mathrm{~L}$ of water was pre-filtered on $100 \mu \mathrm{m}$ mesh to remove metazoans and filtered 139 onto $47 \mathrm{~mm} 2 \mu \mathrm{m}$ Isopore TTTP membrane filters (Millipore Corp., MA, USA) taken in 3x3 140 replicates. The filters were stored at $-20{ }^{\circ} \mathrm{C}$ in cryovials until DNA extraction. Samples for 141 nutrients were collected as described in Thrane et al. (2014). Concentrations of TP, TOC and 142 total nitrogen were determined using standard techniques (for details, see Thrane et al., 2014).

143 Chemical characteristics of the water (e.g. nutrients, $\mathrm{pH}$ and ionic strength) are the most relevant 144 environmental factors determining changes in plankton community composition. TOC and TP 145 were chosen as proxies in the study design to reveal regional environmental gradients and local 146 nutrient supply variability, respectively. The third variable, conductivity, is directly related to the 147 concentration of ionic solutes, and therefore serves as an indicator of soil depth and landscape 148 productivity that is less affected by local pollution than TP (Ryder, 1982). It is important to take 149 into account that not all predictor variables are completely independent (Thrane et al., 2014). 
150 151

152

153

154

155

156

157

158

159

160

161

162

163

164

165

166

167

168

169

170

171

172

Pearson correlation coefficients for the relationship between TOC and TP was 0.61 , and for TP and conductivity was 0.54 ( $\mathrm{P}<0.00001$; all variables log transformed) (Fig. S1, Table S1).

\subsection{DNA extraction, amplification and 454-sequencing of the ITS2 region}

DNA was extracted from the filters using NucleoSpin ${ }^{\circledR}$ Plant II Kit (Mackerey-Nagel, Düren, Germany) according to the protocol from the manufacturer and quantified using Nanodrop (NanoDrop Technologies Inc, DE, USA). The fungal specific modified forward fITS7a ('A' is inserted instead of 'R' at position 5) and reverse ITS4 primers (Ihrmark et al., 2012; White et al., 1990) were used to amplify ITS2. Fusion primers for 454 pyrosequencing incorporating these sequences were designed according to the protocol by Roche by adding adaptors A and B, a key (TCAG) and 10-bp unique tags (MIDs in Roche technical bulletin 005 - 2009) to the forward and reverse primers, respectively. The fusion primers were used in PCR amplifications performed on a PTC-200 DNA Engine Cycler (BioRad, USA) in 20- $\mu$ l reaction volumes containing $4 \mu \mathrm{l}$ of DNA template (i.e. 5 - $10 \mathrm{ng}$ ), 1x Phusion HF buffer, $0.2 \mathrm{mM}$ dNTPs, $0.25 \mu \mathrm{M}$ of each primer, $0.02 \mathrm{U} / \mu \mathrm{l}$ Phusion HotStart II polymerase (Finnzymes, Vantaa, Finland), 3\% DMSO and $1 \mathrm{mg}$ $\mathrm{ml}^{-1}$ BSA (New England BioLabs, Auckland, New Zealand). The amplification program was as follows: $30 \mathrm{~s}$ at $98{ }^{\circ} \mathrm{C}$, followed by 30 cycles of $10 \mathrm{~s}$ at $98{ }^{\circ} \mathrm{C}, 30 \mathrm{~s}$ at $53{ }^{\circ} \mathrm{C}$ and $30 \mathrm{~s}$ at $72{ }^{\circ} \mathrm{C}$, with a final extension step at $72{ }^{\circ} \mathrm{C}$ for $5 \mathrm{~min}$ before storage at $-20{ }^{\circ} \mathrm{C}$. PCR products were cleaned with a Wizard ${ }$ SV Gel and PCR Clean-Up System (Promega, Madison, WI, USA), using a Sequalprep Normalization Plate (96) Kit (Invitrogen, Paisley, UK) and pooled into equimolar amplicon libraries. Ten samples were sequenced twice (technical replicates) to test for sequencing consistency. The 454 Titanium sequencing of the tagged amplicons was performed using GS FLX Titanium (Lib-A chemistry) at the Norwegian Sequencing Centre at the 
173 University of Oslo (Norway) on 1/2 of a 454 FLX Titanium sequencing plate (454 Life Sciences,

174 Branford, CT, USA). The raw 454 reads with corresponding mapping files were deposited in

175 Dryad (doi:xx.xxxx/dryad.xxxxx).

176

177 2.4. Bioinformatics

178 A total of 434603 (average length $424.7 \mathrm{nt}$ ) reads from 87 samples were quality-filtered, 179 denoised, and processed using QIIME v. 1.8.0 (Caporaso et al., 2010) on the Abel cluster at the 180 University of Oslo. All reads with mismatched forward and/or reverse tags were removed to 181 avoid false positives in amplicon data set (Carlsen et al., 2012). Sequences with length $<200 \mathrm{nt}$ 182 and $>550 \mathrm{nt}$, average Phred quality score of $<25$, mismatches in the tags, homopolymers 183 exceeding $6 \mathrm{nt}$, ambiguous base calls $>1$ and $>1$ mismatch in the primers were discarded. In 184 addition, reads were checked for quality by using a 50-nt sliding window (average quality score $185>25)$ to identify regions of low-sequence quality and truncated to the last good window. The 186 resulting sequences (280 502) were denoised using DeNoiser v. 091 (Reeder and Knight, 2010), 187 as implemented in QIIME v. 1.5.0. ITSx 1.0.11 (Bengtsson-Palme et al., 2013) was used to 188 remove the flanking $5.8 \mathrm{~S}$ and $28 \mathrm{~S}$ rRNA gene fragments for optimal resolution of ITS2 189 clustering and removal of compromised and non-target sequences. As filtering removed most of 190 the partial sequences (83 909), we retained only sequences > 99 nt in length (175 853 reads), as 191 suggested by Tedersoo et al. (2014). Reads were clustered into OTUs using the UCLUST 192 algorithm (Edgar, 2010) with a 97\% similarity threshold. The 97\% similarity cut-off has been 193 widely used to delineate fungal OTUs in most comparable aquatic studies (Duarte et al., 2015; 194 Gutiérrez et al., 2015), although it has been shown that ITS region is not equally variable among 195 five fungal phyla with intraspecific ITS variability ranging from zero to $24.2 \%$ (Nilsson et al., 
2008). Global singletons (OTUs represented by only a single sequence across the entire data set) were considered probable sequencing errors and removed (Kunin et al., 2010; Quince et al., 2009; Tedersoo et al., 2010). The most abundant representative sequence per OTU was selected and subjected to BLAST searches against the NCBI-nr/nt database (version 2.2.29). Taxonomic assignments were made by comparing the representative sequence of each OTU against reference databases NCBI-nr/nt and UNITE v. 7 (unite.ut.ee). For a broad taxonomic annotation, taxonomy was assigned at the level of order and family. When the top BLAST match was to unclassified or uncultured fungus, the top 10 matches (if available) were screened for concordance and if possible, taxonomy was assigned based on the subsequent best hits meeting the minimum thresholds of $>80 \%$ sequence similarity and $>70 \%$ coverage. All those OTUs with best BLAST matches to non-fungal organisms, or a best match with $<80 \%$ sequence similarity and $<70 \%$ coverage to a reference sequence assigned to the kingdom Fungi were discarded as non-fungal or unidentifiable OTUs. OTUs were considered putative chimeras and discarded when matching the two criteria: (i) being identified as chimeric by both UCHIME and PERSEUS (Edgar et al., 2011; Quince et al., 2011) and (ii) having a top BLAST match with $<90 \%$ coverage and $<90 \%$ sequence similarity to a reference sequence assigned to the kingdom Fungi (Mundra et al., 2015). For lower rank taxonomy assignment (i.e. genus level) the RDP Naïve Bayesian rRNA Classifier Version 2.11 against the Warcup Fungal ITS training set 2 with 95\% confidence threshold was used, as suggested by Deshpande et al. (2016).

\subsection{Statistical analyses}

To minimize the effect of abundance measure inconsistencies, community composition analyses were conducted on presence/absence data using Jaccard's dissimilarity index (function vegdist in 
219 vegan package). Downstream statistical analyses were performed in $\mathrm{R}$ version 3.2.2 ( $\mathrm{R}$

220 Development Core Team, 2015) using the package vegan (Oksanen et al., 2013) for multivariate

221 and species richness analyses unless otherwise noted. Rarefaction curves were constructed by

222 applying the rarecurve function in vegan.

223 Ordinations by non-metric multidimensional scaling (NMDS) (Minchin, 1987) were used to 224 describe patterns of variation in fungal OTU composition along the longitudinal gradient. 225 Similarity of NMDS ordinations with two $(\mathrm{k}=2)$ and three $(\mathrm{k}=3)$ dimensions was evaluated by 226 Kendall's rank correlation coefficient $\tau$ between NMDS axes when matching the two criteria: $227|\tau|>0.4$ and the corresponding P-value $<0.05$ (Liu et al., 2008). Since the majority of 228 dissimilarity indices used to estimate $\beta$-diversity across sites can vary due to changes in the other 229 two components ( $\alpha$ - and $\gamma$-diversity), we compared the performance of several distance metrics 230 (Bray-Curtis, Jaccard, Gower and Raup-Crick, as implemented by the 'bray', 'jaccard', 'gower' 231 and 'raup' options for the vegdist function in vegan) on our data set in eight different variations 232 using NMDS ordination $(\mathrm{k}=2)$. Assessment of metrics' validity was done by Procrustes 233 correlation run in 999 permutations (function procrustes in package vegan). In addition, NMDS 234 ordinations were conducted on a subset of the matrix representing ten technical replicates to 235 confirm that sequencing-induced variation was smaller than biological variation in the samples. 236 Permutation-based significance tests by the envfit function were used to fit spatial (longitude, 237 latitude, altitude) and environmental (TOC, TP and conductivity, all log transformed) factors to 238 the NMDS ordination $(\mathrm{k}=2)$. The ordisurf function in vegan was used to fit response variables 239 (TOC and TP, both log transformed) as contour lines to the NMDS ordinations $(\mathrm{k}=2)$. To 240 account for sequencing bias, NMDS was conducted on the subset of lakes (with > 150 total reads 241 and $>10 \mathrm{X}$ coverage; coverage $=$ total reads / OTUs richness per lake) resulting in a data set of 
24230 lakes. A standard Mantel test on geographic location and environmental variables to 243 investigate correlation between lakes (function mantel in vegan) was run using Raup-Crick 244 dissimilarity index between aquatic fungal communities and 999 permutations. Raup-Crick 245 distance is robust to the differences in $\alpha$-diversity than other dissimilarity metrics (Birtel et al., 246 2015; Chase et al., 2011). In addition, partial Mantel test (function mantel.partial in vegan) to 247 analyse solely the effect of local environment (TOC, TP and conductivity, all log transformed; 248 Euclidean distance) by partialing out the effect of space (longitude, latitude and altitude) was 249 done.

250 The aquatic fungal communities were split into core (abundant) and transient (occasional or rare)

251 OTUs based on the position of each OTU within the log-normal species abundance distribution 252 (SAD) using persistence-abundance plots as described in Kostovcik et al. (2015).

254 3. Results

255 3.1. Data characteristics

256 After quality filtering and denoising, 280502 reads of the original 434603 reads were retained. 257 Of these, 175853 reads were > 99 nt long ITS2 sequences. The resulting sequences clustered 258 into 3808 OTUs, of which 1857 had no BLAST hit in the NCBI-nr/nt and UNITE databases, 2591026 matched to non-fungal organisms, and 209 OTUs had poor matches to fungi $(<80 \%$ 260 sequence similarity and $<70 \%$ coverage in the BLAST analysis). All these OTU groups were 261 regarded as non-fungal and discarded, leaving 716 fungal OTUs. Among these, an additional 484 262 were removed as singletons or chimeras, leaving a final, curated dataset of 232 fungal OTUs 263 comprising 18738 reads (4.3\% of the initial reads), including the ten technical replicates used 264 for checking sequencing consistency. The ten technical replicate pairs had more similar OTU 
265 composition than random pairwise comparisons between samples (Fig. S2), demonstrating little

266 influence of biases introduced during PCR and sequencing on community composition measures.

267 After removal of the technical replicates, a total of 16513 sequences (3.8\% of initial raw reads)

268 representing 232 OTUs for the 77 lake samples were used for downstream analyses.

\section{3.2. Total fungal richness}

271 Rarefaction curves of OTU richness for each lake indicated that the total fungal diversity was not

272 recovered in most of the lakes (Fig.2). A significant relationship between richness and

273 sequencing depth was observed (both log transformed; P $<0.001$, Pearson correlation coefficient

$274=0.40)($ Fig. S3). In other words, the ordinations showed structuring by sequencing depth, which

275 likely reflects the under-sampling bias.

276

\section{3.3. Taxonomic fungal diversity}

278 Environmental fungal sequences obtained in our study clustered within the major fungal phyla 279 Ascomycota, Basidiomycota and Chytridiomycota. A very few sequences belonging to 280 Zygomycota were retrieved, and phyla like Cryptomycota and Glomeromycota either had no 281 representation in our data set, or remained unassigned. Representative sequences of the OTUs 282 were subjected to two independent similarity searches. First, we assigned taxonomy against the 283 NCBI nr/nt database containing both identified and unidentified sequences (version 2.2.29). To 284 account for possible misclassification of aquatic fungal sequences by GenBank, taxonomic 285 assignment was also done against the curated, quality-checked fungal ITS sequence database 286 UNITE (version 7), where many of the sequences undergo rigorous filtering and classification to 287 species hypothesis using phylogenetic evaluation (Kõljalg et al., 2013). Finally, a representative 
307 in southern Norway.

308 The closest matches to the following fungal genera were obtained in the present study:

sequence for each OTU was classified using the RDP Naïve Bayesian rRNA Classifier v.2.11 against the Warcup Fungal ITS training set 2 to assign taxonomy below the order level, as suggested by Deshpande et al. (2016). A total of 36 orders of fungi were detected (Tables S2 S3). A total of $44.83 \%$ of the OTUs ( $15.21 \%$ of reads) belonged to Basidiomycota, while the Ascomycota accounted for $25.86 \%$ OTUs (5.43\% of the reads). Chytridiomycota was represented by $20.26 \%$ OTUs ( $63.37 \%$ of the reads), while a small proportion of OTUs $(2.16 \%$, $0.19 \%$ of reads) belonged to Zygomycota, and the remaining $6.90 \%$ OTUs ( $15.81 \%$ of the reads) were not assigned at the phylum level. The 20 most frequently observed OTUs represented $82.23 \%$ total reads (Fig. 3A).

Sequences from Ascomycota matched 12 known orders, whereas Basidiomycota was represented by 20 known orders. The dominant basidiomycete orders were the Agaricales (9.91\% OTUs), Tremellales (4.31\% OTUs), Polyporales (3.88\% OTUs) and Russulales (3.88\% OTUs). The most common ascomycete orders were the Pleosporales (8.62\% OTUs), Helotiales (3.45\% OTUs) and Hypocreales (2.59\% OTUs). The chytrid diversity in the lakes was represented by the orders Rhizophydiales (1.29\% OTUs), Chytridiales (0.86\% OTUs) and Spizellomycetales (0.43\% OTUs). $17.67 \%$ chytrid OTUs remained unclassified at the order level. Zygomycota diversity was exclusively represented by Mortierellales (2.16\% OTUs) (Table S2). Interestingly, OTU 1013 had the closest match (98\% similarity) to the parasitic chytrid strain Rhizophydiales sp. Chy-Lys2009 (FR670788; 4 sites, 120 reads) isolated from Planktothrix-dominated lake Lyseren Cryptococcus (9 OTUs), Cortinarius (8 OTUs), Rhodotorula (5 OTUs), Taphrina (4 OTUs), Exidia (4 OTUs), Microdochium (3 OTUs), Mycena (3 OTUs), Sistotrema (3 OTUs), Leccinium 
311 (3 OTUs), Xylodon (3 OTUs), Alternaria (2 OTUs), Malassezia (2 OTUs), Sporobolomyces (2

312 OTUs), Trichosporon (2 OTUs), Gymnopus (2 OTUs), Ceratobasidium (2 OTUs), Itersonilia (2

313 OTUs), Mastigobasidium (2 OTUs), Heterobasidion (2 OTUs), Peniophora (2 OTUs), Lactarius

314 (2 OTUs), Cladosporium (1 OTU), Exophiala (1 OTU), Chytriomyces (1 OTU),

315 Rhizoclosmatium (1 OTU), Betamyces (1 OTU), Globomyces (1 OTU) and Powellomyces (1

316 OTU). The detailed taxonomic assignments for the final 232 OTUs are presented in Table S3

317 (NCBI/UNITE assignment) and Appendix 1 (RDP Naïve Bayesian Classifier).

3.4. Ecology of aquatic fungi

320 The fungi detected in this study included both presumed resident and transient components of 321 aquatic communities. Fig. 3B shows the partition between core and transient fungal OTUs based 322 on the position of each OTU within the log-normal SAD using persistence-abundance plot 323 according to Kostovcik et al. (2015). Occupancy (number of sites in which each OTU was 324 present) was plotted against persistence (maximum read abundance of each OTU across all sites). 325 The occupancy threshold for the split between core and transient OTU groups was found by 326 minimizing the Akaike Information Criterion (AIC) for the fit of the core group to a log-normal 327 rank-abundance distribution. The identified core community consisted of 9 OTUs (colored dots 328 on the right in Fig. 3B). The remaining OTUs were classified as primarily transient and 329 presumably of terrestrial origin (Fig. 3B, left) based on their taxonomic affinities to well-defined 330 groups of terrestrial fungi. However, it is possible some low abundance and rare aquatic taxa 331 could be captured in the 'transient' category as well. The members of 10 most abundant fungal 332 orders, i.e. members of Rhizophydiales (including unassigned OTUs), Capnodiales, and 333 Tremellales were quantitatively more abundant than expected, whereas Pleosporales, Agaricales, 
334 Malasseziales, Polyporales and Sporidiobolales had a large number of relatively low-abundance 335 OTUs (Fig. 3C).

336 In order to investigate the richness effect on $\beta$-diversity in NMDS analyses, we compared several 337 dissimilarity indices (i.e. Bray-Curtis, Jaccard, Gower and Raup-Crick) on eight subsets of sites 338 (Fig. S4). Jaccard, Raup-Crick and Bray-Curtis dissimilarity indices produced very similar 339 results, with Gower being the least robust metrics. Since NMDS axes for two and three 340 dimensions were highly correlated (NMDS1: $\mathrm{P}<0.00001, \tau=0.89$; NMDS2: $\mathrm{P}<0.00001, \tau=$ 3410.87 , respectively), we will focus on the two first NMDS dimensions. To test the robustness of 342 our NMDS analysis, ordinations based on subsets with minimum total reads from 50 to 150 (38 343 to 64 lakes) were compared (all with Procrustes significance probabilities $=0.001$ on 999 344 permutations, and Procrustes correlations $=0.73-0.99)$, suggesting that the conservative choice 345 of > 150 reads is probably valid for much wider subsets (Fig. S5). The NMDS analysis 346 performed for the subset of studied lakes (with $>150$ total reads and $>10 \mathrm{X}$ coverage) found 347 significant correlation between aquatic fungal community composition and longitude $(\mathrm{P}=0.001)$ 348 as well as local environmental factors (TOC $(\mathrm{P}=0.005), \mathrm{TP}(\mathrm{P}=0.001)$ and conductivity $(\mathrm{P}=$ 349 0.002), all log transformed) (Fig. 4A, 4B and Table S4).

350 Community dissimilarity increased significantly with geographical distance (Mantel correlation $351=0.20, \mathrm{P}=0.001$ on 999 permutations) and local environment (Mantel correlation $=0.17, \mathrm{P}=$ 3520.002 on 999 permutations). The significant effect of the local environment (TOC, TP and 353 conductivity, all log transformed) persisted after spatial adjustment by a partial Mantel test 354 (Mantel correlation $=0.12, \mathrm{P}=0.03$ on 999 permutations). 
358 Fungi in freshwater have varying ecological roles, e.g. as decomposers, pathogens or parasites of 359 sponges, fish, crustaceans, algae or other fungi (Gleason et al., 2008; Ishida et al., 2015; Kagami 360 et al., 2007; Wurzbacher et al., 2010) and can occur as residents (adapted to aquatic environments) or transients (occurring in water fortuitously) (Shearer et al., 2007). The relatively

362 low number of fungal OTUs (232) detected in our study may be due to the exclusion of most 363 basidiomycetes and zygomycetes, to the lower species diversity of plant hosts in aquatic habitats, 364 to environmental restrictions on growth of fungi in the water column, or dominance of fungal 365 groups (i.e. Cryptomycota) that are underrepresented in ITS2 databases (Bärlocher and Boddy, 2016; Shearer et al., 2007). Moreover, it is difficult to compare OTU numbers between studies

367 due to the fact that bioinformatics processing parameters can significantly impact OTU detection 368 and richness estimates (Gihring et al., 2012; Kunin et al., 2010; Quince et al., 2009; Schloss, 369 2010). Richards et al. (2015) suggest that the DNA extraction protocols used are likely biased 370 against the recovery of fungal sequences (in particular, filamentous forms with robust cell walls).

371 However, assuming the low levels of diversity recovered here do represent a species-poor 372 community, the drivers responsible for this may be similar to those in marine ecosystems as, for 373 example, low nutrient levels, absence of substrates for fungal cell attachment, and the dominance 374 of free-floating single-celled plankton in the photic zone (Richards et al., 2012). Fungal 375 community composition varied both with longitude and local environmental factors suggesting 376 that fungi, like protists, respond to local and metacommunity scale productivity gradients 377 (Ptacnik et al., 2010). TOC (i.e. the sum of suspended particulate and dissolved organic matter) 378 will probably reflect resource availability for osmotrophs and saprotrophs, and may, as such, 379 influence the fungal community composition. In our study we aimed at selecting boreal lakes 
380

381

382

383

384

385

386

387

388

389

390

391

392

393

394

395

396

397

398

399

400

401

402

within the TOC and TP range typical for this region. Since dissolved organic matter (which constitutes $>90 \%$ of TOC in these lakes) contains a small, but non-zero, amount of phosphorus, there will be a weak, but unavoidable covariation between TOC and TP. We excluded lakes with particularly high TOC since these will not be equally available across the spatial gradient (i.e. brown-water lakes are less common in the west). We also excluded lakes with particularly high TP since these typically reflect local pollution rather than regional trends. We deliberately constrained the climatic variation in our study by making the longitudinal gradient three times longer $(750 \mathrm{~km})$ than the latitudinal (Khomich et al., in press). With this study design we find a strong longitudinal signal of the same magnitude as in earlier studies with non-molecular methods (Hessen et al., 2006; Ptacnik et al., 2010). The results of Mantel test suggest that adjacent lakes tend to be compositionally more similar. Moreover, effects of the local environment on aquatic fungal communities were still present after partialing out spatial factors.

The fungi detected in this study included a large fraction of putatively terrestrial taxa and taxa known to occur in both terrestrial and aquatic ecosystems. This concurs with other studies that have detected both resident and transient components of fungal communities in aquatic ecosystems (Gutiérrez et al., 2015; Zhang et al., 2015), although conclusions regarding the terrestrial or aquatic status of the OTUs recovered here must be drawn with caution, as high confidence taxonomic assignments to the genus and species level are severely hampered by under-populated reference databases, and low abundance or rare aquatic species may be classed as 'transient' components of the community using SAD analyses. However, based on their taxonomic affinity to known groups of terrestrial fungi, the putative terrestrial fungal OTUs likely are fungal structures that have been washed into aquatic habitats where they are not active contributors to the community, but still can be detected. In general, overlap between species in 
403 freshwater and terrestrial habitats can be high compared to between marine and freshwater, or 404 marine and terrestrial habitats (Shearer et al., 2007; Zhang et al., 2015), reflecting the 405 evolutionary history of aquatic fungi, which has been suggested to include multiple transitions 406 from terrestrial to aquatic forms (Vijaykrishna and Hyde, 2006).

407 The most abundant OTUs were taxonomically assigned to Chytridiomycota (Fig. 3A and 3B), 408 whose members are known to be saprobes on a large variety of substrates and parasites of 409 phytoplankton, zooplankton, fungi and invertebrates (Gleason et al., 2008; Kagami et al., 2014; 410 Sime-Ngando, 2012). Although the pre-filtering of water samples on $100 \mu \mathrm{m}$ mesh could 411 potentially exclude some filamentous fungi or fungi attached to phytoplankton and thus reduce 412 the detected chytrid diversity, our findings are largely in agreement with literature sources 413 (Jobard et al., 2012; Lefèvre et al., 2012) and confirm the importance of basal fungal groups in 414 aquatic food webs (Kagami et al., 2014; Sime-Ngando, 2012). These planktonic fungi are strictly 415 dependent on surfaces and, therefore, are closely associated with all types of aquatic organisms 416 (e.g. algae, copepods) and most likely also lake snow particles (Tang et al., 2006; Wurzbacher et 417 al., 2010). However, most surveys on freshwater parasite dynamics are focused on hosts (e.g. 418 cyanobacteria and diatoms) (Sime-Ngando, 2012), with several studies reporting chytrid 419 parasitism as an important ecological factor for determining abundance of the filamentous 420 cyanobacterium Planktothrix in lakes in southern Norway (Kyle et al., 2015; Rohrlack et al., 421 2015). Interestingly, one OTU recovered here had the closest match (98\% similarity) to the 422 chytrid strain Rhizophydiales sp. Chy-Lys2009 (FR670788) which was isolated from the 423 Planktothrix-dominated lake Lyseren in southern Norway. According to Sønstebø and Rohrlack 424 (2011), this chytrid strain showed high infectious capability for Planktothrix sp., but failed to 425 infect other filamentous cyanobacteria. 
428 Members of Basidiomycota occurred frequently in the surface waters of oligotrophic lakes, but 429 with lower abundance than those of the Ascomycota. Our results are not in concordance with 430 previous studies in which early diverging fungal lineages were found to be the dominant fungal 431 forms in freshwater habitats (Lefèvre et al., 2012). Although Dikarya is the dominant fungal 432 group in marine environments (Bass et al., 2007; Edgcomb et al., 2011; Tisthammer et al., 2016; 433 Zhang et al., 2015), 'basal' lineages are thought to dominate in fresh and brackish waters 434 (Lefèvre et al., 2012; Richards et al., 2012; Shearer et al., 2007). In particular, Ascomycota 435 (including aquatic hyphomycetes), Chytridiomycota and other true fungi comprise most of the 436 documented freshwater fungal diversity (Bärlocher and Boddy, 2016; Shearer et al., 2007). The 437 inconsistency between our findings and the prevailing opinion in the literature may partially be 438 explained by possible primer bias, since the primers used in our data set have not been evaluated 439 against members of the basal fungal lineages (Ihrmark et al., 2012), and were designed to 440 amplify Dikarya. Alternatively, the high diversity of Basidiomycota detected in our study may 441 reflect detection of the transient component of the fungal community. Basidiomycota are 442 dominant fungal community components in terrestrial environments (Buée et al., 2009) and the 443 most commonly identified orders of Basidiomycota in this study were common terrestrial 444 lineages like Agaricales, Polyporales and Russulales. Their occurrence in these aquatic 445 ecosystems is likely due to transient introductions through transport of allochthonous organic 446 material (Bärlocher, 2016) and deposition of wind-dispersed spores. In addition, a significant 447 proportion of Basidiomycota detected in the studied lakes belonged to ectomycorrhizal fungal 448 orders, e.g. Agaricales and Thelephorales. As with invertebrates and plants (Green et al., 2008), a 

466 2006). may explain their presence in the surface waters of lakes (Hyde and Soytong, 2008). Among Ascomycota the most commonly detected orders were Pleosporales, Helotiales and Hypocreales, all of which include members with aquatic lifestyles. However, it is difficult to determine if these fungi represent transient or resident members of the fungal community. Members of the Pleosporales and Hypocreales are known to be lignicolous (growing on submerged woody debris) in both aquatic and terrestrial habitats (Hyde et al., 2016; Hyde et al., 2013; Maharachchikumbura et al., 2015; Wijayawardene et al., 2014), so it is unclear whether the detected OTUs represent transient contributions of spores of terrestrial species or the signature of resident populations of fungi decomposing material in the littoral zone. Similarly, leaf-degrading fungi from ascomycete genera Aureobasidium, Cladosporium, Alternaria and Phoma detected in this study play an initial role in emergent macrophyte decomposition (Wurzbacher et al., 2010) and cannot be easily classed as resident or transient community members. Some ascomycetes categorized as freshwater fungi have also been reported from other habitats (e.g. terrestrial and marine), indicating possible ubiquitous distribution of some species, which is consistent with evolutionary reconstructions that support a terrestrial origin for both freshwater ascomycetes and marine ascomycetes (Kodsueb et al., 2016; Vijaykrishna and Hyde,

\subsection{Yeast-like forms}

The majority of higher fungi occurring in aquatic environments have unicellular yeast growth forms. Interestingly, yeasts may account for more biomass and diversity than filamentous fungi in these systems. Yeast-like growth forms are expected to be more abundant in freshwater 
472 environments than in seawater, comprising < 100 cells/l in unpolluted lakes versus $<10$ cells/l in 473 open ocean waters (Hagler and Ahearn, 1987).

474 Yeast-like forms detected in our study belonged to the basidiomycetous orders Tremellales, 475 Sporidiobolales, Leucosporidiales, Malasseziales and Trichosporonales (altogether 10.34\% 476 OTUs), and ascomycetous orders Taphrinales, Saccharomycetales and Dothideales (altogether $4773.44 \%$ OTUs). Similarly, basidiomycetous yeasts often constitute the bigger fraction of the total 478 yeast population in oligotrophic oceanic waters (Nagahama, 2006). Earlier it was assumed that 479 yeasts are transients washed in from the phylloplane or the littoral zone, but there is now clear 480 evidence for their more or less permanent residence in open waters. Members of Tremellales 481 (Cryptococcus species) have previously been reported in lake surface waters (Rosa et al., 1995; 482 Van Uden and Ahearn, 1963; Wurzbacher et al., 2010). An opportunistic black yeast pathogen 483 genus Aureobasidium (Dothideales) found in our study is considered to enter aquatic 484 environments with plant material, and the red yeast Sporobolomyces spp. (Sporidiobolales) is 485 similarly introduced by the fallen leaves of terrestrial plants (Libkind et al., 2009; Nagahama, 486 2006). The genus Rhodotorula (Sporidiobolales) includes ubiquitous saprophytic yeasts isolated 487 from different aquatic habitats and invertebrates (Nagahama et al., 2003), and it was 488 unsurprisingly detected widely in our samples. Two OTUs belonging to Malasseziales were 489 found in our study systems. Culture-independent studies of fungi from environmental samples 490 show that Malassezia (Malasseziales) is a cosmopolitan lipophilic yeast widely distributed in 491 deep-sea sediments, hydrothermal vents, stony corals, fish guts, Antarctic soils and in the 492 exoskeleton of soil nematodes (Amend, 2014; Bass et al., 2007; Edgcomb et al., 2011; Gao et al., 493 2008). It is currently unclear whether the diversity of Malassezia-like organisms may reflect 494 similar ecological diversity with trophic strategies ranging from saprotrophy to biotrophy 
495 (Amend, 2014). One OTU was assigned to the black yeasts genus Exophiala (Chaetothyriales), 496 which includes ubiquitous, opportunistic pathogens causing both superficial and systemic 497 mycoses in marine and freshwater fish (e.g. halibut, salmon, cod), although it can also be isolated 498 from substrates such as soil, sediments, decaying wood and plant material (Gjessing et al., 2011; 499 Overy et al., 2015). Similarly, other known yeast-like pathogens recovered in this study closely 500 related to terrestrial fungi and associated with disease in aquatic environment include 501 Trichosporon, Taphrina, Ustilago and Exophiala (Higgins, 2000; Richards et al., 2012).

\subsection{Chytridiomycota, Cryptomycota and Zygomycota}

504 Our results show a significant fraction of unclassified fungal sequences in surface waters of lakes

505 (up to $16 \%$ of total reads) suggesting that surface waters of oligotrophic lakes can be a source of 506 unknown fungal diversity. Richards et al. (2012) hypothesize that aquatic environments host a 507 significant number of unclassified novel groups branching below the Dikarya radiation, thus 508 reflecting an ancient transition from aquatic to terrestrial ecosystems. Among the currently 509 recognized basal fungal lineages, chytrid fungi were detected in high abundances in our study, 510 and are known to be ubiquitous in aquatic environments, both as saprotrophs and obligate 511 parasites of phytoplankton (James et al., 2006; Kagami et al., 2007; Shearer et al., 2007; Voigt et 512 al., 2013).

513 The three known Chytridiomycota orders, namely Rhizophydiales, Chytridiales and 514 Spizellomycetales, were all detected, with a large fraction of sequences matching poorly to the 515 available ITS2 references. Importantly, identification of Chytridiomycota increasingly relies on a 516 combination of both ultrastructure and molecular data suggesting a polyphyletic nature for many 517 chytrid genera (Grossart et al., 2016; Karpov et al., 2014; Letcher et al., 2008a; Letcher et al., 
518 2012; Letcher et al., 2008b; Letcher et al., 2008c). Possible mismatches in reference databases

519 limit our ability to infer the ecological role of these chytrid fungi based solely on environmental

520 sequences. In addition, the great majority of Chytridiomycota have not been cultured and studied,

521 and are thus classified as 'uncultured' in sequence databases (Grossart et al., 2016).

522 However, not only parasitic, but also saprotrophic fungal lifestyles can be of ecological

523 relevance. For example, saprotrophic Chytridiomycota are commonly found on pollen, which 524 occurs in huge quantities in lakes, particularly during the clear-water phase in spring when 525 organic matter and nutrients are low (Wurzbacher et al., 2014). It is unclear whether the 526 Chytridiomycota detected in this study represent saprotrophic or parasitic taxa. We hypothesize 527 that both functional groups may be present in the aquatic environment occupying narrow 528 ecological niches (Gleason et al., 2008; Rasconi et al., 2011).

529 Representatives of a single order in Zygomycota, Mortierellales, were detected in this study at 530 low frequency and abundance. Zygomycota are known to be relatively rare in aquatic habitats 531 (Shearer et al., 2007) and are most commonly saprobic soil-inhabiting fungi on decaying organic 532 material (Wagner et al., 2013) that most likely enter the aquatic environment transiently with 533 wind or soil particles.

534 Cryptomycota are known to occur in freshwater environments, as well as in marine and soil 535 ecosystems, however the group was notably absent from our samples. While the group may in 536 fact be rare in surface waters, we hypothesize that our inability to detect Cryptomycota in the 537 studied lakes is more likely attributable to primer bias, as the primers used have not been tested 538 against the group, or as a result of the paucity of ITS2 sequences for this group in public 539 databases (<1\% sequences in the UNITE database) which may cause Cryptomycota sequences 540 to be incorrectly identified as non-fungal or classified only to the Kingdom level. 
543 The fungal-specific primer (fITS7a) used in this study, was tested for members of the

544 Ascomycota, Basidiomycota, and Zygomycota, and focused on the exclusion of the plant 545 Kingdom (Ihrmark et al., 2012). As a result, the primer's specificity for other groups, including 546 basal fungal lineages and animals, remains largely unknown. The high proportion of OTUs in 547 this study that remained unassigned (48.77\%) or matched non-target organisms (26.94\%) (e.g. 548 Chlorophyta, Cryptophyta and Ciliophora) suggests that the primer combination fITS7a/ITS4 is 549 not fungal-specific in aquatic environments. This substantially reduced the effective sequencing 550 depth for fungal taxa, and as a result, the full diversity of the aquatic fungal communities was not 551 recovered in this study (see Fig.2).

552 The high proportion of unidentified sequences in our data set may reflect poor ITS database 553 coverage, as some of the taxa that were expected to be dominant (Chytridiomycota and 554 Cryptomycota) have very little ITS information available for them. An alternative would be to 555 use the more conserved LSU/SSU regions which have the advantages of inferring higher-level 556 phylogenetic relationships and identifying novel fungal lineages, though they cannot 557 discriminate between closely related fungal species. Therefore, a combination of several gene 558 markers achieves a higher and more reliable phylogenetic inference, as has recently been 559 established for Chytridiomycota (Grossart et al., 2016; Lefèvre et al., 2012; Letcher et al., 2008c). 560 Few studies have shown that both ITS and LSU regions provided comparable accuracy in 561 estimating fungal diversity and taxonomic assignments to the genus level (Brown et al., 2014; 562 Porras-Alfaro et al., 2014). Nevertheless, the ITS approach remains useful when targeting well- 
563 defined taxonomic groups where species level OTU identification is important (Brown et al., 564 2014; Richards et al., 2012).

565 Another concern is that detection of rare species (or OTUs) can be particularly difficult in low 566 density populations, as would be expected in surface water fungal communities. In agreement 567 with previous surveys (Lefèvre et al., 2012), a majority of the taxonomically assigned true fungi 568 detected in our study were only found in few lakes suggesting they are likely rare. Therefore, an 569 intense sampling of aquatic fungal communities should be considered to improve the exploration 570 of rare taxa which may play an important role by becoming dominant in response to 571 environmental changes, as well as representing a novel source of diversity. Moreover, an

572 alternative could be to sample monthly or seasonally to track the presence of particular aquatic 573 species, as well as to obtain a better understanding of the main factors regulating pelagic fungal 574 communities, as has recently been shown for small eukaryotes in freshwater ecosystems (Mangot 575 et al., 2013; Simon et al., 2015).

576

577 4.6. Concluding remarks

578 In conclusion, our results suggest that surface waters of oligotrophic lakes harbour many fungal 579 taxa, but many of these seem to be transient and likely transported from the surrounding 580 terrestrial environment by wind, water and/or migratory birds. Importantly, there are several 581 limitations to our study that can inform future surveys of freshwater fungi using amplicon 582 sequencing. First, fITS7a/ITS4 performed poorly as a fungal-specific primer combination in an 583 aquatic environment. Non-target amplification hampered sequencing depth to the extent that we 584 were unable to capture the total fungal diversity. Secondly, the scarcity of taxonomic references 585 for basal fungal lineages in public ITS2 sequence databases creates difficulties in identifying 
586 OTUs below the phylum level, such that targeting more conserved gene regions may be more 587 appropriate under some circumstances.

588

589 Acknowledgements

590 We thank the COMSAT field sampling crew, especially Dag. O. Hessen, Johnny Håll, Marcia 591 Kyle, Robert Ptacnik, and Jan-Erik Thrane, for their efforts.

592

$593 \quad$ Funding

594 This study has been supported financially by the Department of Biosciences, University of Oslo 595 and by the Research Council of Norway (contract Miljø2015/196336 "Biodiversity, community 596 saturation and ecosystem function in lakes" (COMSAT)).

597 


\section{References}

Amend, A. (2014) From dandruff to deep-sea vents: Malassezia-like fungi are ecologically hyper-diverse. PLoS Pathogens 10: e1004277.

Bass, D., Howe, A., Brown, N., Barton, H., Demidova, M., Michelle, H. et al. (2007) Yeast forms dominate fungal diversity in the deep oceans. Proceedings of the Royal Society of London B: Biological Sciences 274: 3069-3077.

Bengtsson - Palme, J., Ryberg, M., Hartmann, M., Branco, S., Wang, Z., Godhe, A. et al. (2013) Improved software detection and extraction of ITS1 and ITS2 from ribosomal ITS sequences of fungi and other eukaryotes for analysis of environmental sequencing data. Methods in Ecology and Evolution 4: 914-919. Birtel, J., Walser, J.-C., Pichon, S., Bürgmann, H., and Matthews, B. (2015) Estimating bacterial diversity for ecological studies: methods, metrics, and assumptions. PloS one 10: e0125356.

Blaalid, R., Carlsen, T., Kumar, S., Halvorsen, R., Ugland, K.I., Fontana, G., and Kauserud, H. (2012) Changes in the root - associated fungal communities along a primary succession gradient analysed by 454 pyrosequencing. Molecular Ecology 21: 1897-1908.

Botnen, S., Vik, U., Carlsen, T., Eidesen, P.B., Davey, M.L., and Kauserud, H. (2014) Low host specificity of root - associated fungi at an Arctic site. Molecular Ecology 23: 975-985.

Brown, S.P., Rigdon-Huss, A.R., and Jumpponen, A. (2014) Analyses of ITS and LSU gene regions provide congruent results on fungal community responses. Fungal Ecology 9: 65-68.

Buée, M., Reich, M., Murat, C., Morin, E., Nilsson, R.H., Uroz, S., and Martin, F. (2009) 454 Pyrosequencing analyses of forest soils reveal an unexpectedly high fungal diversity. New Phytologist 184: 449-456.

Bärlocher, F. (2016) Aquatic hyphomycetes in a changing environment. Fungal Ecology 19: 14-27.

Bärlocher, F., and Boddy, L. (2016) Aquatic fungal ecology - How does it differ from terrestrial? Fungal Ecology 19: 5-13.

Caporaso, J.G., Kuczynski, J., Stombaugh, J., Bittinger, K., Bushman, F.D., Costello, E.K. et al. (2010) QIIME allows analysis of high-throughput community sequencing data. Nature Methods 7: 335-336.

Carlsen, T., Aas, A.B., Lindner, D., Vrålstad, T., Schumacher, T., and Kauserud, H. (2012) Don't make a mista $(\mathrm{g})$ ke: is tag switching an overlooked source of error in amplicon pyrosequencing studies? Fungal Ecology 5: 747-749.

Chase, J.M., Kraft, N.J., Smith, K.G., Vellend, M., and Inouye, B.D. (2011) Using null models to disentangle variation in community dissimilarity from variation in a - diversity. Ecosphere 2: 1-11.

Chauvet, E., Cornut, J., Sridhar, K.R., Selosse, M.-A., and Bärlocher, F. (2016) Beyond the water column: aquatic hyphomycetes outside their preferred habitat. Fungal Ecology 19: 112-127.

Comeau, A.M., Vincent, W.F., Bernier, L., and Lovejoy, C. (2016) Novel chytrid lineages dominate fungal sequences in diverse marine and freshwater habitats. Scientific Reports 6: 30120.

Deshpande, V., Wang, Q., Greenfield, P., Charleston, M., Porras-Alfaro, A., Kuske, C.R. et al. (2016) Fungal identification using a Bayesian classifier and the Warcup training set of internal transcribed spacer sequences. Mycologia 108: 1-5.

Duarte, S., Bärlocher, F., Trabulo, J., Cássio, F., and Pascoal, C. (2015) Stream-dwelling fungal decomposer communities along a gradient of eutrophication unraveled by 454 pyrosequencing. Fungal Diversity 70: 127-148.

Duarte, S., Bärlocher, F., Pascoal, C., and Cássio, F. (2016) Biogeography of aquatic hyphomycetes: Current knowledge and future perspectives. Fungal Ecology 19: 169-181.

Edgar, R.C. (2010) Search and clustering orders of magnitude faster than BLAST. Bioinformatics 26: 24602461. 
Edgar, R.C., Haas, B.J., Clemente, J.C., Quince, C., and Knight, R. (2011) UCHIME improves sensitivity and speed of chimera detection. Bioinformatics 27: 2194-2200.

Edgcomb, V.P., Beaudoin, D., Gast, R., Biddle, J.F., and Teske, A. (2011) Marine subsurface eukaryotes: the fungal majority. Environmental Microbiology 13: 172-183.

Gao, Z., Li, B., Zheng, C., and Wang, G. (2008) Molecular detection of fungal communities in the Hawaiian marine sponges Suberites zeteki and Mycale armata. Applied and Environmental Microbiology 74: 6091-6101.

Gihring, T.M., Green, S.J., and Schadt, C.W. (2012) Massively parallel rRNA gene sequencing exacerbates the potential for biased community diversity comparisons due to variable library sizes. Environmental Microbiology 14: 285-290.

Gjessing, M.C., Davey, M., Kvellestad, A., and Vrålstad, T. (2011) Exophiala angulospora causes systemic inflammation in Atlantic cod Gadus morhua. Diseases of Aquatic Organisms 96: 209-219.

Gleason, F.H., Kagami, M., Lefevre, E., and Sime-Ngando, T. (2008) The ecology of chytrids in aquatic ecosystems: roles in food web dynamics. Fungal Biology Reviews 22: 17-25.

Graça, M.A., Hyde, K., and Chauvet, E. (2016) Aquatic hyphomycetes and litter decomposition in tropical-subtropical low order streams. Fungal Ecology 19: 182-189.

Green, A.J., Jenkins, K., Bell, D., Morris, P., and Kingsford, R. (2008) The potential role of waterbirds in dispersing invertebrates and plants in arid Australia. Freshwater Biology 53: 380-392.

Grossart, H.-P., Wurzbacher, C., James, T.Y., and Kagami, M. (2016) Discovery of dark matter fungi in aquatic ecosystems demands a reappraisal of the phylogeny and ecology of zoosporic fungi. Fungal Ecology 19: 28-38.

Gutiérrez, M.H., Galand, P.E., Moffat, C., and Pantoja, S. (2015) Melting glacier impacts community structure of Bacteria, Archaea and Fungi in a Chilean Patagonia fjord. Environmental Microbiology 17: 3882-3897.

Hagler, A.N., and Ahearn, D. (1987) Ecology of aquatic yeasts. The Yeasts 2: 181-205.

Hassett, B., Ducluzeau, A., Collins, R., and Gradinger, R. (2016) Spatial distribution of aquatic marine fungi across the western Arctic and sub - Arctic. Environmental microbiology.

Hassett, B., and Gradinger, R. (2016) Chytrids dominate arctic marine fungal communities. Environmental microbiology 18: 2001-2009.

Henriksen, A., Skjelvåle, B.L., Mannio, J., Wilander, A., Harriman, R., Curtis, C. et al. (1998) Northern European lake survey, 1995: Finland, Norway, Sweden, Denmark, Russian Kola, Russian Karelia, Scotland and Wales. Ambio 27: 80-91.

Hessen, D.O., Faafeng, B.A., Smith, V.H., Bakkestuen, V., and Walseng, B. (2006) Extrinsic and intrinsic controls of zooplankton diversity in lakes. Ecology 87: 433-443.

Higgins, R. (2000) Bacteria and fungi of marine mammals: a review. The Canadian Veterinary Journal 41: 105-116.

Hortal, J., Nabout, J.C., Calatayud, J., Carneiro, F.M., Padial, A., Santos, A. et al. (2014) Perspectives on the use of lakes and ponds as model systems for macroecological research. $J$ Limnol 73: 46-60. Hyde, K., and Soytong, K. (2008) The fungal endophyte dilemma. Fungal Diversity 33: 163-173. Hyde, K.D., Jones, E.G., Liu, J.-K., Ariyawansa, H., Boehm, E., Boonmee, S. et al. (2013) Families of dothideomycetes. Fungal Diversity 63: 1-313.

Hyde, K.D., Fryar, S., Tian, Q., Bahkali, A.H., and Xu, J. (2016) Lignicolous freshwater fungi along a northsouth latitudinal gradient in the Asian/Australian region; can we predict the impact of global warming on biodiversity and function? Fungal Ecology 19: 190-200.

Ihrmark, K., Bödeker, I.T., Cruz-Martinez, K., Friberg, H., Kubartova, A., Schenck, J. et al. (2012) New primers to amplify the fungal ITS2 region-evaluation by 454-sequencing of artificial and natural communities. FEMS Microbiology Ecology 82: 666-677. 
Ishida, S., Nozaki, D., Grossart, H.P., and Kagami, M. (2015) Novel basal, fungal lineages from freshwater phytoplankton and lake samples. Environmental Microbiology Reports 7: 435-441.

James, T.Y., Letcher, P.M., Longcore, J.E., Mozley-Standridge, S.E., Porter, D., Powell, M.J. et al. (2006) A molecular phylogeny of the flagellated fungi (Chytridiomycota) and description of a new phylum (Blastocladiomycota). Mycologia 98: 860-871.

Jobard, M., Rasconi, S., Solinhac, L., Cauchie, H.M., and Sime - Ngando, T. (2012) Molecular and morphological diversity of fungi and the associated functions in three European nearby lakes. Environmental Microbiology 14: 2480-2494.

Jones, E.G., Hyde, K.D., and Pang, K.-L. (2014) Introduction. In Freshwater fungi: and fungal-like organisms. Jones, E.B.G., K.D. Hyde, K.-L Pang (ed). Berlin, Germany: De Gruyter, pp. 1-22. Kagami, M., de Bruin, A., Ibelings, B.W., and Van Donk, E. (2007) Parasitic chytrids: their effects on phytoplankton communities and food-web dynamics. Hydrobiologia 578: 113-129.

Kagami, M., Miki, T., and Takimoto, G. (2014) Mycoloop: chytrids in aquatic food webs. Front Microbiol 5: 166.

Karpov, S., Kobseva, A., Mamkaeva, M., Mamkaeva, K., Mikhailov, K., Mirzaeva, G., and Aleoshin, V. (2014) Gromochytrium mamkaevae gen. \& sp. nov. and two new orders: Gromochytriales and Mesochytriales (Chytridiomycetes). Persoonia: Molecular Phylogeny and Evolution of Fungi 32: 115-126. Khomich, M., Kauserud, H., Logares, R., Rasconi, S., and Andersen, T. (2016) Planktonic protistan communities in lakes along a large-scale environmental gradient. FEMS Microbiology Ecology: fiw231.

Kodsueb, R., Lumyong, S., McKenzie, E., Bahkali, A., and Hyde, K. (2016) Relationships between terrestrial and freshwater lignicolous fungi. Fungal Ecology 19: 155-168.

Kõljalg, U., Nilsson, R.H., Abarenkov, K., Tedersoo, L., Taylor, A.F., Bahram, M. et al. (2013) Towards a unified paradigm for sequence - based identification of fungi. Molecular Ecology 22: 5271-5277.

Kostovcik, M., Bateman, C.C., Kolarik, M., Stelinski, L.L., Jordal, B.H., and Hulcr, J. (2015) The ambrosia symbiosis is specific in some species and promiscuous in others: evidence from community pyrosequencing. The ISME journal 9: 126-138.

Kuehn, K.A. (2016) Lentic and lotic habitats as templets for fungal communities: traits, adaptations, and their significance to litter decomposition within freshwater ecosystems. Fungal Ecology 19: 135-154.

Kunin, V., Engelbrektson, A., Ochman, H., and Hugenholtz, P. (2010) Wrinkles in the rare biosphere: pyrosequencing errors can lead to artificial inflation of diversity estimates. Environmental Microbiology 12: 118-123.

Kyle, M., Haande, S., Ostermaier, V., and Rohrlack, T. (2015) The red queen race between parasitic chytrids and their host, Planktothrix: A test using a time series reconstructed from sediment DNA. PloS one 10: e0118738.

Lefèvre, E., Letcher, P.M., and Powell, M.J. (2012) Temporal variation of the small eukaryotic community in two freshwater lakes: emphasis on zoosporic fungi. Aquatic Microbial Ecology 67: 91-105.

Leibold, M.A., Holyoak, M., Mouquet, N., Amarasekare, P., Chase, J., Hoopes, M. et al. (2004) The metacommunity concept: a framework for multi - scale community ecology. Ecol Lett 7: 601-613.

Letcher, P.M., Powell, M.J., Barr, D.J., Churchill, P.F., Wakefield, W.S., and Picard, K.T. (2008a) Rhizophlyctidales-a new order in Chytridiomycota. Mycological Research 112: 1031-1048.

Letcher, P.M., Powell, M.J., and Viusent, M.C. (2008b) Rediscovery of an unusual chytridiaceous fungus new to the order Rhizophydiales. Mycologia 100: 325-334.

Letcher, P.M., Vélez, C.G., Barrantes, M.E., Powell, M.J., Churchill, P.F., and Wakefield, W.S. (2008c) Ultrastructural and molecular analyses of Rhizophydiales (Chytridiomycota) isolates from North America and Argentina. Mycological Research 112: 759-782.

Letcher, P.M., Powell, M.J., and Picard, K.T. (2012) Zoospore ultrastructure and phylogenetic position of Phlyctochytrium aureliae Ajello is revealed (Chytridiaceae, Chytridiales, Chytridiomycota). Mycologia 104: 410-418. 
Libkind, D., Gadanho, M., van Broock, M., and Sampaio, J.P. (2009) Cystofilobasidium lacus-mascardii sp. nov., a basidiomycetous yeast species isolated from aquatic environments of the Patagonian Andes, and Cystofilobasidium macerans sp. nov., the sexual stage of Cryptococcus macerans. International journal of systematic and evolutionary microbiology 59: 622-630.

742 Liu, H., Økland, T., Halvorsen, R., Gao, J., Liu, Q., Eilertsen, O., and Bratli, H. (2008) Gradient analyses of areas, S and SW China. Sommerfeltia 32: 1-196.

Maharachchikumbura, S.S., Hyde, K.D., Jones, E.G., McKenzie, E.H., Huang, S.-K., Abdel-Wahab, M.A. et al. (2015) Towards a natural classification and backbone tree for Sordariomycetes. Fungal Diversity 72 : 199-301.

Mangot, J.F., Domaizon, I., Taib, N., Marouni, N., Duffaud, E., Bronner, G., and Debroas, D. (2013) Short - term dynamics of diversity patterns: evidence of continual reassembly within lacustrine small eukaryotes. Environmental Microbiology 15: 1745-1758.

Minchin, P.R. (1987) An evaluation of the relative robustness of techniques for ecological ordination. Vegetatio 69: 89-107.

Monchy, S., Sanciu, G., Jobard, M., Rasconi, S., Gerphagnon, M., Chabé, M. et al. (2011) Exploring and quantifying fungal diversity in freshwater lake ecosystems using rDNA cloning/sequencing and SSU tag pyrosequencing. Environmental Microbiology 13: 1433-1453.

Mundra, S., Halvorsen, R., Kauserud, H., Müller, E., Vik, U., and Eidesen, P.B. (2015) Arctic fungal communities associated with roots of Bistorta vivipara do not respond to the same fine - scale edaphic gradients as the aboveground vegetation. New Phytologist 205: 1587-1597.

Nagahama, T., Hamamoto, M., Nakase, T., and Horikoshi, K. (2003) Rhodotorula benthica sp. nov. and Rhodotorula calyptogenae sp. nov., novel yeast species from animals collected from the deep-sea floor, and Rhodotorula lysiniphila sp. nov., which is related phylogenetically. International Journal of Systematic and Evolutionary Microbiology 53: 897-903.

Nagahama, T. (2006) Yeast biodiversity in freshwater, marine and deep-sea environments. In Biodiversity and ecophysiology of yeasts. Rosa, C.A., P. Gábor (ed). Heidelberg, Germany: Springer, pp. 241-262.

Nilsson, R.H., Kristiansson, E., Ryberg, M., Hallenberg, N., and Larsson, K.-H. (2008) Intraspecific ITS variability in the kingdom Fungi as expressed in the international sequence databases and its implications for molecular species identification. Evolutionary Bioinformatics Online 4: 193-201.

Oksanen, J., Blanchet, F., Kindt, R., Legendre, P., Minchin, P., O'Hara, R. et al. (2013) Vegan: community ecology package. $R$ package version 2.0.7. http://cran.r-project.org/.

Overy, D.P., Groman, D., Giles, J., Duffy, S., Rommens, M., and Johnson, G. (2015) Exophiala angulospora Causes Systemic Mycosis in Atlantic Halibut: a Case Report. Journal of Aquatic Animal Health 27: 12-19.

Porras-Alfaro, A., Liu, K.-L., Kuske, C.R., and Xie, G. (2014) From genus to phylum: large-subunit and internal transcribed spacer rRNA operon regions show similar classification accuracies influenced by database composition. Applied and Environmental Microbiology 80: 829-840.

Ptacnik, R., Solimini, A.G., Andersen, T., Tamminen, T., Brettum, P., Lepistö, L. et al. (2008) Diversity predicts stability and resource use efficiency in natural phytoplankton communities. Proc Natl Acad Sci USA 105: 5134-5138.

Ptacnik, R., Andersen, T., Brettum, P., Lepistö, L., and Willén, E. (2010) Regional species pools control community saturation in lake phytoplankton. Proceedings of the Royal Society of London B: Biological Sciences 277: 3755-3764.

Quince, C., Lanzén, A., Curtis, T.P., Davenport, R.J., Hall, N., Head, I.M. et al. (2009) Accurate determination of microbial diversity from 454 pyrosequencing data. Nature Methods 6: 639-641.

Quince, C., Lanzen, A., Davenport, R.J., and Turnbaugh, P.J. (2011) Removing noise from pyrosequenced amplicons. BMC Bioinformatics 12: 38. 
R Development Core Team (2015). R: A Language and Environment for Statistical Computing. R Foundation for Statistical Computing, Vienna, Austria. Available at: http://www.R-project.org Rasconi, S., Jobard, M., and Sime-Ngando, T. (2011) Parasitic fungi of phytoplankton: ecological roles and implications for microbial food webs. Aquatic Microbial Ecology 62: 123-137.

Rasconi, S., Niquil, N., and Sime - Ngando, T. (2012) Phytoplankton chytridiomycosis: community structure and infectivity of fungal parasites in aquatic ecosystems. Environmental microbiology 14: 21512170.

Reeder, J., and Knight, R. (2010) Rapidly denoising pyrosequencing amplicon reads by exploiting rankabundance distributions. Nature Methods 7: 668-669.

Richards, T.A., Jones, M.D., Leonard, G., and Bass, D. (2012) Marine fungi: their ecology and molecular diversity. Annual Review of Marine Science 4: 495-522.

Richards, T.A., Leonard, G., Mahé, F., del Campo, J., Romac, S., Jones, M.D. et al. (2015) Molecular diversity and distribution of marine fungi across 130 European environmental samples. Proceedings of the Royal Society B: Biological Sciences 282: 20152243.

Rohrlack, T., Haande, S., Molversmyr, A.., and Kyle, M. (2015) Environmental conditions determine the course and outcome of phytoplankton chytridiomycosis. PloS one 10: e0145559.

Rosa, C.A., Resende, M.A., Barbosa, F.A., Morais, P.B., and Franzot, S.P. (1995) Yeast diversity in a mesotrophic lake on the karstic plateau of Lagoa Santa, MG-Brazil. Hydrobiologia 308: 103-108.

Ryder, R. (1982) The morphoedaphic index-use, abuse, and fundamental concepts. Transactions of the American Fisheries Society 111: 154-164.

Schloss, P.D. (2010) The effects of alignment quality, distance calculation method, sequence filtering, and region on the analysis of 16S rRNA gene-based studies. PLoS Computational Biology 6: e1000844.

Shearer, C.A., Descals, E., Kohlmeyer, B., Kohlmeyer, J., Marvanová, L., Padgett, D. et al. (2007) Fungal biodiversity in aquatic habitats. Biodiversity and Conservation 16: 49-67.

Sime-Ngando, T. (2012) Phytoplankton chytridiomycosis: fungal parasites of phytoplankton and their imprints on the food web dynamics. Frontiers in microbiology 3: 361.

Simon, M., López-García, P., Deschamps, P., Moreira, D., Restoux, G., Bertolino, P., and Jardillier, L. (2015) Marked seasonality and high spatial variability of protist communities in shallow freshwater systems. The ISME Journal 9: 1941-1953.

Soininen, J. (2012) Macroecology of unicellular organisms-patterns and processes. Environ Microbiol Rep 4: 10-22.

Solheim, A.L., Rekolainen, S., Moe, S.J., Carvalho, L., Phillips, G., Ptacnik, R. et al. (2008) Ecological threshold responses in European lakes and their applicability for the Water Framework Directive (WFD) implementation: synthesis of lakes results from the REBECCA project. Aquatic Ecology 42: 317-334.

Sønsteb $\varnothing$, J.H., and Rohrlack, T. (2011) Possible implications of chytrid parasitism for population subdivision in freshwater cyanobacteria of the genus Planktothrix. Applied and environmental microbiology 77: 1344-1351.

Tang, K.W., Hutalle, K.M.L., and Grossart, H.-P. (2006) Microbial abundance, composition and enzymatic activity during decomposition of copepod carcasses. Aquatic Microbial Ecology 45: 219-227.

Tedersoo, L., Nilsson, R.H., Abarenkov, K., Jairus, T., Sadam, A., Saar, I. et al. (2010) 454 Pyrosequencing and Sanger sequencing of tropical mycorrhizal fungi provide similar results but reveal substantial methodological biases. New Phytologist 188: 291-301.

Tedersoo, L., Bahram, M., Põlme, S., Kõljalg, U., Yorou, N.S., Wijesundera, R. et al. (2014) Global diversity and geography of soil fungi. Science 346: 1256688.

Thrane, J.-E., Hessen, D.O., and Andersen, T. (2014) The absorption of light in lakes: Negative impact of dissolved organic carbon on primary productivity. Ecosystems 17: 1040-1052.

Thüs, H., Aptroot, A., and Seaward, M. (2014) Freshwater lichens. In Freshwater fungi and fungal-like organisms. Jones, E.B.G., K.D. Hyde , K.-L. Pang (ed). Berlin, Germany: De Gruyter, pp. 333-358. 
834 Tisthammer, K.H., Cobian, G.M., and Amend, A.S. (2016) Global biogeography of marine fungi is shaped 835 by the environment. Fungal Ecology 19: 39-46.

836 Van Uden, N., and Ahearn, D. (1963) Occurrence and population densities of yeast species in a fresh837 water lake. Antonie van Leeuwenhoek 29: 308-312.

838 Vijaykrishna, D., and Hyde, K.D. (2006) Inter-and intra stream variation of lignicolous freshwater fungi in 839 tropical Australia. Fungal Diversity 21: 203-224.

840 Voigt, K., Marano, A.V., and Gleason, F.H. (2013) Ecological and Economical Importance of Parasitic 841 Zoosporic True Fungi. In Agricultural Applications, 2nd Edition, The Mycota XI. Kempken, F. (ed). 842 Heidelberg, Germany: Springer, pp. 243-270.

843 Voronin, L. (2014) Terrigenous micromycetes in freshwater ecosystems (review). Inland Water Biology 7: 844 352-356.

845 Wagner, L., Stielow, B., Hoffmann, K., Petkovits, T., Papp, T., Vágvölgyi, C. et al. (2013) A comprehensive 846 molecular phylogeny of the Mortierellales (Mortierellomycotina) based on nuclear ribosomal DNA. 847 Persoonia: Molecular Phylogeny and Evolution of Fungi 30: 77-93.

848 White, T.J., Bruns, T., Lee, S., and Taylor, J. (1990) Amplification and direct sequencing of fungal 849 ribosomal RNA genes for phylogenetics. PCR protocols: a guide to methods and applications 18: 315-322. 850 Wijayawardene, N.N., Crous, P.W., Kirk, P.M., Hawksworth, D.L., Boonmee, S., Braun, U. et al. (2014) 851 Naming and outline of Dothideomycetes-2014 including proposals for the protection or suppression of 852 generic names. Fungal Diversity 69: 1-55.

853 Wurzbacher, C., Rösel, S., Rychła, A., and Grossart, H.-P. (2014) Importance of saprotrophic freshwater 854 fungi for pollen degradation. Plos One 9: e94643.

855 Wurzbacher, C.M., Bärlocher, F., and Grossart, H.-P. (2010) Fungi in lake ecosystems. Aquatic Microbial 856 Ecology 59: 125-149.

857 Zhang, T., Wang, N.F., Zhang, Y.Q., Liu, H.Y., and Yu, L.Y. (2015) Diversity and distribution of fungal 858 communities in the marine sediments of Kongsfjorden, Svalbard (High Arctic). Scientific Reports 5: 14524. 


\section{$860 \quad$ Figure legends}

861 Fig. 1. A map of sampled lakes $(n=77)$. The mountain ridge extends $\mathrm{S}-\mathrm{N}$ around $8{ }^{\circ} \mathrm{E}$. The

862 subset of lakes $(n=30)$ used in the NMDS ordination is indicated by dark red colour.

863

864 Fig. 2. Rarefaction curves for 77 sampled lakes describing the number of fungal OTUs as a 865 function of the number of reads.

866

867 Fig. 3. A) The relative abundance of top 20 fungal OTUs. The identity number of the respective 868 OTU is shown below the bars. Colours represent the fungal phyla. B) Core (right) and transient 869 (left) aquatic community members based on discontinuity in persistence/abundance distribution 870 of fungal OTUs. Dashed line represents the threshold between transient and core groups, 871 identified by minimizing the AIC of a log-normal rank-abundance fit to the core group. C) Top 87210 most abundant fungal orders.

873

874 Fig. 4. Nonmetric multidimensional scaling (NMDS) plot of the subset of lakes $(n=30)$ scaled 875 by OTU richness, coloured by longitude with contour lines indicating change in A) TOC and B) 876 TP (both log transformed).

877 
878 Fig. 1. A map of sampled lakes $(n=77)$. The mountain ridge extends $S-N$ around $8{ }^{\circ} \mathrm{E}$. The 879 subset of lakes $(\mathrm{n}=30)$ used in the NMDS ordination is indicated by dark red colour.

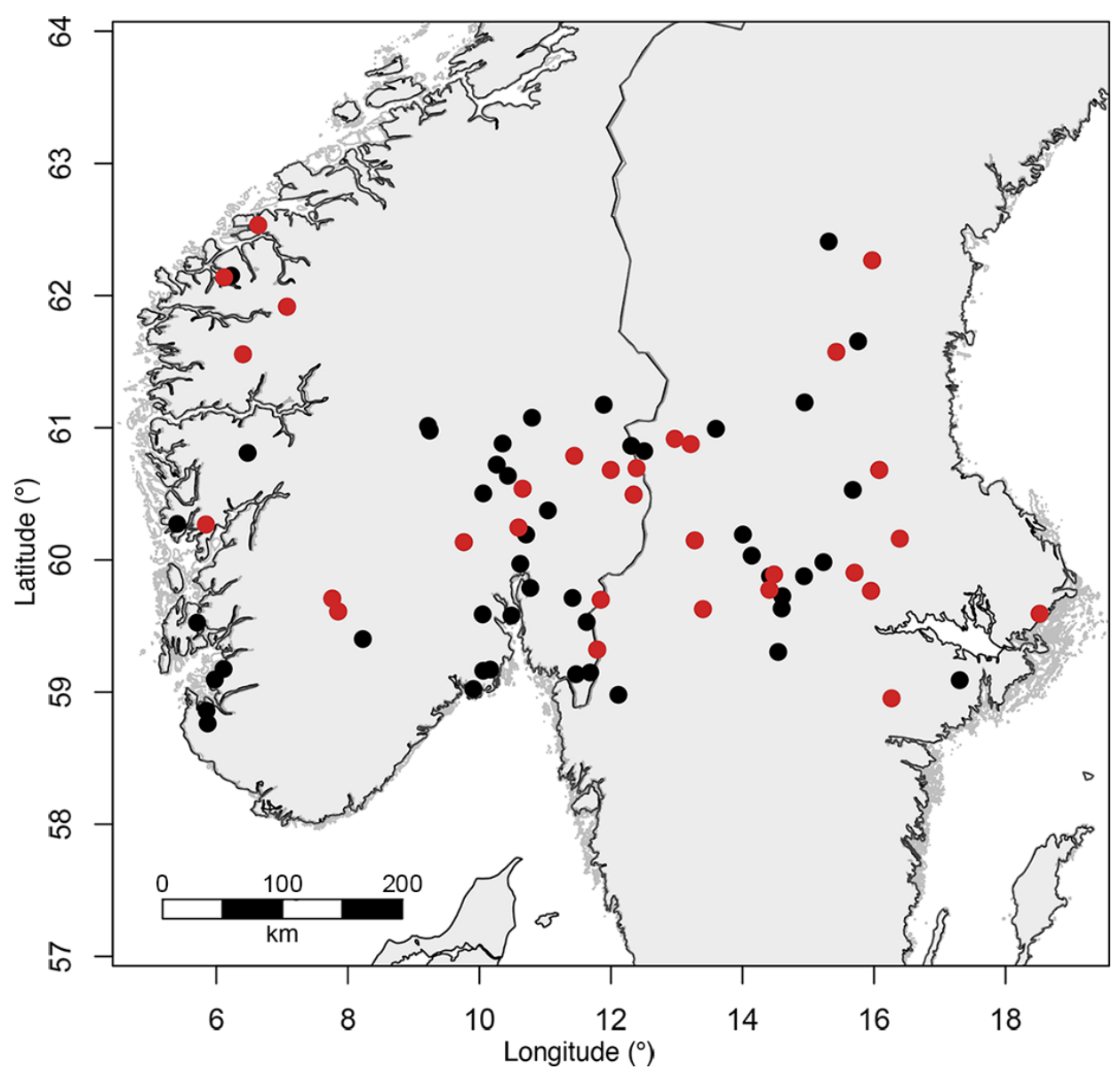


883 Fig. 2. Rarefaction curves for 77 sampled lakes describing the number of fungal OTUs as a 884 function of the number of reads.

885

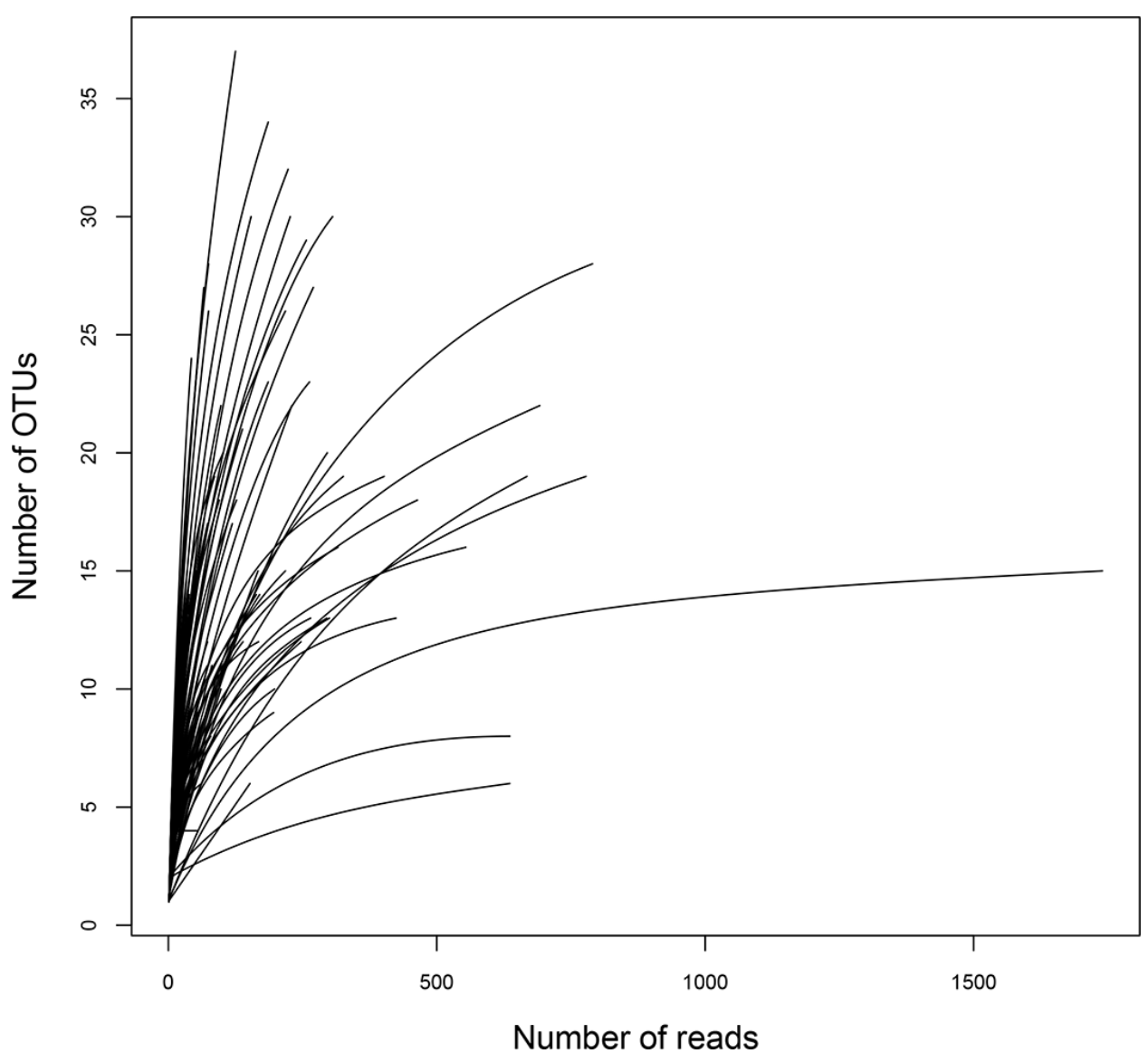

887 
Fig. 3. A) The relative abundance of top 20 fungal OTUs. The identity number of the respective OTU is shown below the bars.

889 Colours represent the fungal phyla. B) Core (right) and transient (left) aquatic community members based on discontinuity in 890 persistence/abundance distribution of fungal OTUs. Dashed line represents the threshold between transient and core groups, identified 891 by minimizing the AIC of a log-normal rank-abundance fit to the core group. C) Top 10 most abundant fungal orders.

892
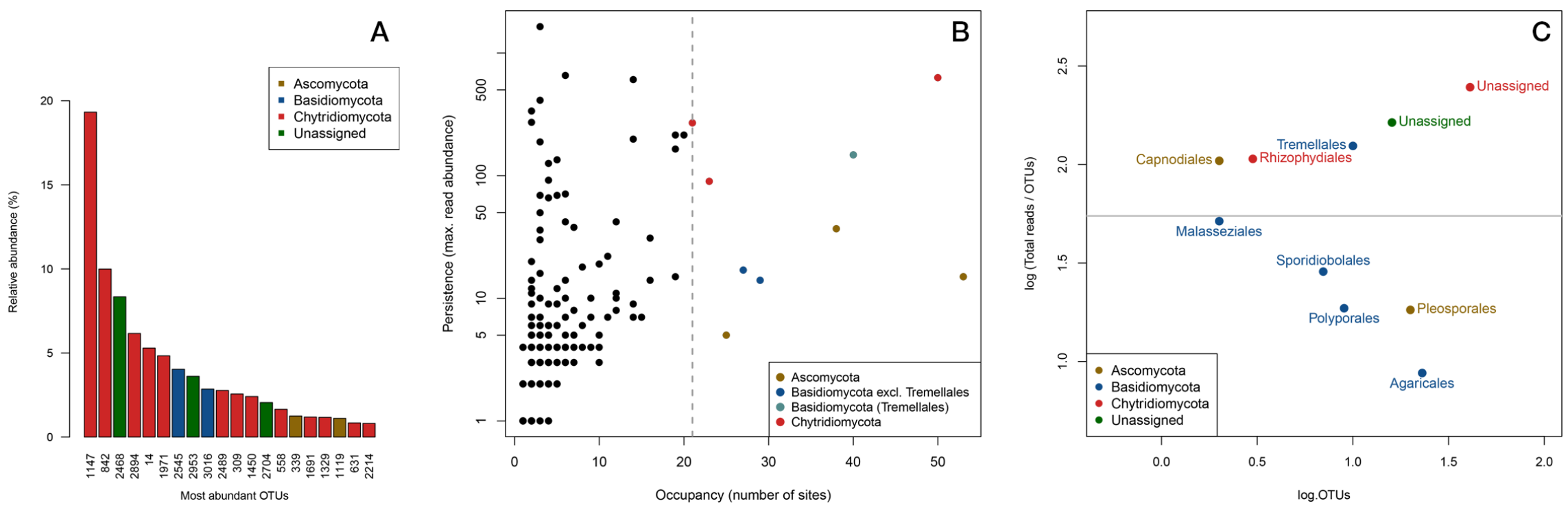
894 Fig. 4. Nonmetric multidimensional scaling (NMDS) plot of the subset of lakes $(\mathrm{n}=30)$ scaled by OTU richness, coloured by 895 longitude with contour lines indicating change in A) TOC and B) TP (both log transformed).

896
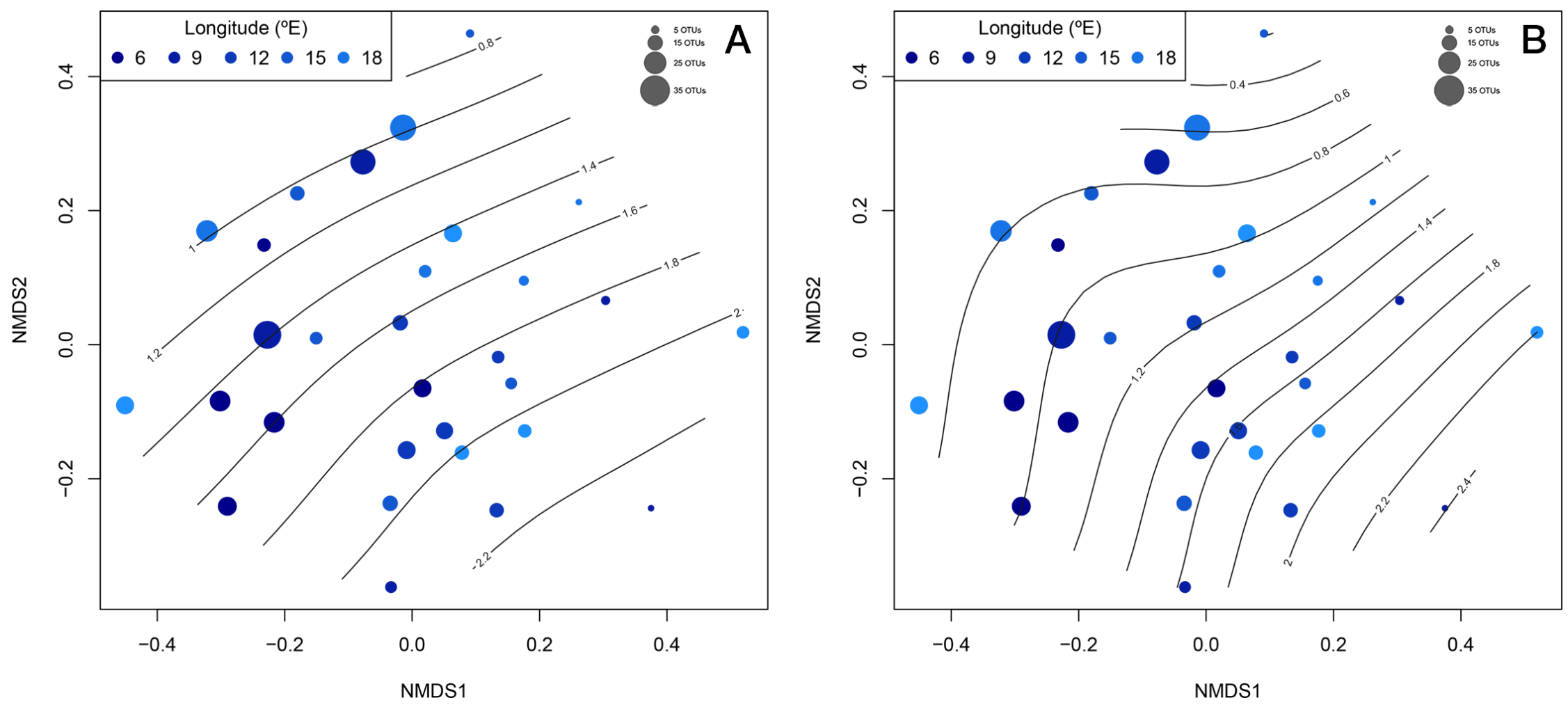